\title{
Petite introduction à une lecture de Gyroscope de Michel Butor
}

\author{
Henri Desoubeaux
}

pour Maxime Godard

\section{Les 2 moitiés}

Comme Transit, le Génie du lieu, 4, quatre ans plus tôt, Gyroscope, publié chez Gallimard en 1996, est fait de deux moitiés tête-bêche1. L'une, intitulée "Porte chiffres », est ornée

\footnotetext{
1 Il s'agit d'un livre grand format à l'italienne, alors que tous les volumes précédents de la série, au même format à l'exception du premier, sont à la française. Par là même, Gyroscope se détache des volumes précédents un peu comme le pouce se détache des autres doigts de la main. Du reste, le livre subira une véritable métamorphose lors de sa republication dans les OEuvres
} 
du mot "GRAVITATIONS », l'autre, intitulée « Entrée lettres », est sommée du mot «REVOLUTIONS». Par ce dispositif, l'auteur entend évoquer les deux hémisphères terrestres et la rotation de la Terre (le titre lui-même y renvoie avec le gyroscope de Foucault) suggérée ici par le mouvement même que le lecteur est amené à faire subir au volume pour en poursuivre l'exploration.

\section{Les 4 canaux}

Chaque moitié est divisée en quatre "canaux ». Chaque double page du livre tout entier présente ainsi quatre colonnes sur lesquelles se développent les Programmes. À propos de ces " canaux », il est bon de se souvenir, me semble-t-il, du bouletwagon du roman de Jules Verne, Autour de la Lune (il en sera question dans le programme Ciel), dont les quatre hublots - un à "droite», un autre "sur l'autre face du projectile, un autre dans le dôme qui le terminait, un quatrième enfin au milieu du culot inférieur» (Verne, p. 30) — permettent l'exploration visuelle de l'espace dans lequel se déplacent les trois héros verniens. Autrement dit, ces quatre canaux n'explorent pas des espaces si différents qu'ils ne puissent communiquer entre eux, qu'ils ne participent d'un même espace plus large. C'est pourquoi le lecteur, outre la voie du suivi de chacun des programmes, est également invité au «zapping » : «Peu à peu on ne pourra s'en empêcher. L'œil, tout en lisant un programme, sera attiré par quelque mot ou formule dans la

complètes aux éditions de la différence en octobre 2008. En effet, il y est publié de façon singulière puisque sur deux volumes, les volumes VII et VIII. La «Porte chiffres» sur le premier, l'«Entrée lettres» sur le second. Cette étude renvoie à l'édition de 1996. 
colonne d'à côté. On percevra accord ou dissonances. On voyagera dans deux ou plusieurs pays à la fois. » (Gyroscope, «Porte chiffres », p. 42, canal 2).

\section{Les 8 programmes}

Observatoire : C5-105, L50-134, L133-195, C137-199

Ciel : C7-43, L64-114, C156-198

Avatar : C8-26 et L28-48

Voyant : C10-40, L40-62, C96-112, L173-199, C127-135, L117131

Cathay : L5-127, C42-164, L53-115, L136-198, C107-175, C99125, L136-198, C114-154

Pyramide : L7-51 et C44-94

Minotaure: L8-24/L24-38 ; C45-59/C59-77/C79-97 ; L129151/L153-171 et C166-200

Vision : L10-26, C28-42, L116-134, C177-197

\section{Les 33 plages}

Plage s'entend ici par l'espace occupé de façon ininterrompue par un programme sur telle ou telle partie d'un canal.

2 plages : Avatar et Pyramide

3 plages : Ciel

4 plages : Observatoire, Minotaure et Vision 
6 plages : Voyant

8 plages : Cathay

\section{La succession des plages}

Le plus souvent, les plages se suivent de $C$ en $L$ ou de $L$ en $C$ (quatre programmes commencent en C, quatre autres commencent en L), autrement dit alternent. Exceptions : Observatoire (L50-134, L133-195) et Cathay. Dans le cas de Minotaure, les quatre plages se subdivisent en huit sections; ainsi, la première plage comprend deux sections qui se lisent sur le même canal l'une à la suite de l'autre sans interruption.

\section{Les tableaux récapitulatifs}

Outre la «table des tables » et les deux «tables» - Canaux lettres (cette dernière comporte trois erreurs ${ }^{2}$ ) et Canaux chiffres - , on trouve aussi une «table des stèles» intitulée « Mémoires », et une autre, intitulée «table des sources ». Pour la première, il s'agit d'une liste d'auteurs : peintres, écrivains, musiciens à qui est dédiée telle ou telle partie («à la mémoire de ... »). On y compte 33 noms, c'est-à-dire autant que de plages. N'y figurent pas cependant les noms du Titien, du Tintoret, de Charles Perrault et de Hugo von Hofmannsthal, qui appartiennent au programme Minotaure. Cela du fait que ce programme est composé de quatre plages, mais aussi de huit sections qui, toutes, possèdent un dédicataire. Pour la seconde,

\footnotetext{
${ }^{2} \mathrm{Au}$ lieu de « Ciel 1 », il faut lire «Vision $1 »$; au lieu de « Cathay 5 », il faut lire « Cathay 7 » et, au lieu de « Cathay 3 », il faut lire « Cathay 4 ».
} 
la table des sources, elle met en évidence une des caractéristiques de l'art de Michel Butor et qui est la citation : "Tous les mots ont déjà été employés; toutes les phrases sont ombres, variations, résurgences d'auteurs parfois nombreux. » (Gyroscope, «Entrée lettres», p. 197, canal C) Enfin, d'autres tableaux existent qui s'intitulent « récapitulation » et qui sont, contrairement aux précédents réunis de part et d'autre de l'axe central, éparpillés dans l'ensemble du volume.

\section{Observatoire}

C5-105 (4), L50-134 (A), L133-195 (C), C137-199 (3)

Dans les sous-titres des programmes, j'ai mis en romain les textes de la «Porte chiffres » et en italique ceux de l'« Entrée lettres ». Entre parenthèses, sous le titre du programme, après la pagination, figurent les canaux.

1 Récitatifs d'Elseneur (à la mémoire de Christian Dotremont)

2 Dialogues d'Elseneur (à la mémoire de Karen Blixen) 3 Trios d'Elseneur1 (à la mémoire de Samuel Taylor Coleridge) 4 Trios d'Elseneur 2 (à la mémoire de Ludvig Holberg)

Dans ce programme, le quatre s'affirme tout particulièrement. Non seulement nous avons quatre plages de texte, mais le début indique : "La scène est divisée en quatre niveaux... Sur le sol quatre édifices, etc. » La table des sources, par ailleurs, indique quatre sources: "Andersen, Kepler, Shakespeare et Tycho-Brahé ». On remarquera, cependant, que ce sont trois titres qui annoncent les textes de ce programme, le troisième étant «Trios d'Elseneur ». Du reste, ce sont trois 
tableaux récapitulatifs qui ponctuent le programme, un à la fin de chaque texte.

L'aire géographique observée, si l'on peut dire, est le nord de l'Europe et, plus particulièrement, le Danemark et même la ville d'Elseneur et, de là, bien sûr, le ciel. Ce programme communique donc naturellement avec le programme suivant.

«Récitatifs d'Elseneur» est extrait de Elseneur, suite dramatique, paru en 1979 avec une préface de Roger Francillon, à laquelle nous renvoyons pour l'analyse de la pièce. Le navire et l'astronef du programme suivant sont déjà à l'œuvre. Dans cette partie, sur les 112 quatrains d'Elseneur, 90 sont repris ou, pour quelques-uns, réécrits, mais apparaissent toujours associés deux à deux sur la même page, si bien que nous avons à faire ici plutôt à des huitains. Les trois fois quatre personnages de la pièce sont repris, mais le chœur a disparu bien que le texte dit par lui soit conservé. L'ensemble est divisé en quatre sections marquées par une couleur distincte comme pour les deux autres textes de ce programme. Il s'agit donc assez largement d'une réécriture de son propre texte à laquelle Butor procède.

Pour la partie « Dialogues d'Elseneur », l'auteur puise à la même source, mais selon un mode propre puisque les dialogues présents sont empruntés aussi bien aux duos de la pièce originelle qu'à ses trios et intègrent parfois des strophes isolées. Les éléments du texte originel semblent ainsi tourner comme les cercles d'une sphère armillaire et sans doute une telle comparaison n'a jamais été aussi bien justifiée. Sur les 74 quatrains présents ici, certains sont nouveaux relativement à la première partie et, contrairement à ce qui se passe dans cette 
dernière, toujours l'italique alterne avec le romain. Nous avons en quelque sorte deux quatrains enchâssés l'un dans l'autre.

Dans les deux "Trios d'Elseneur », ce sont 84 quatrains qui sont présents, dont quelques-uns sont nouveaux par rapport aux parties précédentes si bien que c'est presque tout le texte originel qui est repris dans l'ensemble de ce programme et repris un peu plus de deux fois. La disposition sur la page, cependant, a changé et ce sont seulement six vers qui apparaissent à chaque page, répartis entre trois « acteurs ». À droite du canal, en italique, rappel du programme Ciel, comme pour souligner davantage encore la parenté étroite de ces deux programmes.

\section{Ciel}

C7-43 (3), L64-114 (B), C156-198 (2)

1 Franchir l'espace 1 Autrefois ou les cieux (à la mémoire de Camille Flammarion) 2 Franchir l'espace 2 Aujourd'hui ou les astres (à la mémoire de Lucien de Samosate)

3 Franchir l'espace 3 Demain ou les mondes (à la mémoire de H. G. Wells)

Ce texte, le seul qui s'étale sur trois plages, fait apparaître une composante du même type que dans "Picasso-Labyrinthe » du programme Minotaure, à savoir un ensemble de citations d'une même œuvre qui court tout au long des pages, non en haut comme dans le texte précité, mais en bas, comme pour lui répondre. Il s'agit des Entretiens sur la pluralité des mondes de Fontenelle. L'ouvrage est divisé en six «soirs». Les citations 
incluses dans "Autrefois ou les cieux» appartiennent au "premier soir $»^{3}$. Celles qui sont incluses dans « Aujourd'hui ou les astres » appartiennent aux «second», «troisième » et surtout « quatrième » soirs. Enfin, celles incluses dans « Demain ou les mondes » appartiennent essentiellement au « cinquième soir ». On notera que la citation du " sixième soir », qui achève le programme, renvoie à la Chine à travers l'ouvrage écrit en latin (Abrégé des Annales de la Chine), tout comme à la fin du programme Voyant, l'auteur, à propos du film Le fantôme de l'enfant marcheur, mentionne là encore la Chine: "Je devais l'accompagner en Chine au mois d'octobre... Ce n'est que cinq ans plus tard, en 1993, que je suis allé pour la première fois en Chine. » (Gyroscope, «Entrée lettres », p. 125, canal C) On voit donc par là que le programme Cathay occupe une place particulièrement importante puisque plusieurs programmes convergent vers lui.

Dans « Autrefois », c'est tout d'abord à travers Description de San Marco, un livre de 1963, que se fait l'évocation de la Création et donc des jours de la semaine, mais c'est plus généralement toutes les correspondances qui ont été établies au cours des siècles entre les métaux, les planètes, les dieux, les couleurs, les signes du zodiaque, les vertus théologales et cardinales, etc. que nous pouvons appréhender au fur et à mesure que sont évoqués parallèlement la découverte de l'Amérique (à travers les mémoires de Christophe Colomb), les systèmes de Copernic, de Tycho-Brahé, les découvertes de

\footnotetext{
3 Pour faciliter la lecture, outre la place occupée de manière précise sur la page, l'ensemble des citations de chacune de ces trois plages est encadré des mots en italique : «Échos d'un château ... », au début, d'une part, et « Échos », successivement, « du règne ...», « d'un jardin ...» et « d'une planète ...», à la fin, d'autre part.
} 
Galilée, de Kepler, de Herschel, de Halley, le tout reposant sur le texte, "premier soir », de Fontenelle. Nous n'avons pas encore véritablement quitté la Terre. Le navire symbolise ou emblématise cette partie comme l'astronef symbolise ou emblématise la seconde.

«Aujourd'hui » est dédié à la mémoire de Lucien de Samosate, auteur de l'Histoire véritable, qui narre notamment un voyage sur la Lune. La Lune dont il est d'abord question dans ce texte est évoquée plus spécialement à travers la description du mont Copernic, qu'on trouve dans Autour de la Lune de Jules Verne (1966, p. 169). Puis l'on passe au Soleil. Puis aux planètes Vénus et Mars, puis à la ceinture d'astéroïdes avec ses « astrescités » avant d'aborder les planètes géantes, Jupiter, Saturne et, dans la même page, Uranus, Neptune et, enfin, la lointaine Pluton. C'est donc le système solaire tout entier que nous venons d'explorer à bord de l'astronef butorien, le texte de Butor communiquant avec celui de Fontenelle.

Dans «Demain», le mouvement vers le lointain (nous quittons non seulement le système solaire, mais la Voie lactée pour un au-delà toujours plus lointain) s'accompagne d'un mouvement rétrograde qui nous permet de remonter le temps. Ainsi, par exemple, les mots « quasar » et «premières cellules » voisinent sur la même page. Tout progrès de la connaissance est bidimensionnel. L'astronomie est sœur de l'archéologie, dont il sera question dans d'autres programmes. Le texte de Fontenelle accompagne celui de Butor. Emblème : une surface d'inscription de cinq rangées de rectangles qui tournent chacune dans le sens inverse de celui de ses voisines. 


\section{Avatar}

C8-26 (2) et L28-48 (A)

1 Encore Angkor 1 (à la mémoire de Pierre Loti) 2 Encore Angkor 2 (à la mémoire d'André Breton)

Comme le programme Pyramide, ce programme ne comprend que deux parties : «Encore Angkor 1 » (C8-C26) et «Encore Angkor 2» (L28-L48).

Le texte est en réalité composé de celui paru dans Angkor silencieux avec des photographies de Philippe Gras, en 1988, et repris dans Avant-goût III l'année suivante, et de quelques fragments de "La pluie à Angkor » paru, lui, dans les Cahiers $d u$ Chemin ${ }^{4}$ en janvier 1968 et repris dans le second Génie du lieu, Où, en 1971. Ce sont donc vingt ans qui séparent ces deux éléments, dont l'un est en romain et l'autre en italique dans ces pages.

\section{Voyant}

C10-40 (1), L40-62 (B), C96-112 (2),

L173-199 (D), C127-135 (3), L117-131 (C)

1 Hallucinations simples, premier cahier (à la mémoire du capitaine Frédéric Rimbaud)

\footnotetext{
4 « La boue à Séoul / la pluie à Angkor », Les Cahiers du chemin, n 2 , janvier 1968, p. 48-78. Deux textes qui seront repris également, avant leur entrée dans Où, dans Claude Book-Senninger and Jack Kolbert, L'Art de Michel Butor, New York, Oxford University Press, 1970, p. 107-119 et 122-138.
} 
2 Hallucinations simples, deuxième cahier (à la mémoire de M. C. V. Rimbaud)

3 Hallucinations simples, troisième cahier (à la mémoire de Frédéric Rimbaud)

4 Tournant autour d'Arthur Rimbaud 1 (suite) (à la mémoire de Vitalie-Marie Rimbaud)

5 Tournant autour d'Arthur Rimbaud 2 (à la mémoire de Vitalie-Jeanne Rimbaud)

6 Tournant autour d'Arthur Rimbaud 3 (à la mémoire d'Isabelle Rimbaud)

Le programme Voyant contient non seulement le texte Hallucinations simples ${ }^{5}$, qui s'étend sur un peu plus que les deux premiers «cahiers », mais aussi un historique de ce texte sur le restant du troisième. La suite, "Tournant autour d'Arthur Rimbaud", poursuit en fait cet historique, donne des indications précises sur l'évolution du texte au fur et à mesure de ses publications successives, mentionne les Improvisations sur Rimbaud de 1989 aussi bien que le film Le Fantôme de l'enfant marcheur, court métrage de $25 \mathrm{mn}$ réalisé avec JeanMarie Le Sidaner et William Mimouni en 1988. Elle introduit aussi un entretien avec Jean-Marie Le Sidaner, paru initialement dans la revue Flache en mai 1988 et qui s'étale ici sur les cinquième et sixième plages. Enfin, le programme se termine sur le poème " Outre-Harrar », qui date du 19 mars 1991 (donc écrit pour le centenaire de la mort du poète) et qu'on

5 Cf. «Hallucinations simples», dans Avant-goût II, 1987, ainsi que Hallucinations simples, 1989. 
retrouvera dans le recueil postérieur, L'Horticulteur itinérant (p. 121).

Une remarque doit être faite sur l'emplacement de ces six plages. En effet, la quatrième plage intervient du point de vue de la pagination après les cinquième et sixième plages.

\section{Cathay}

L5-127 (D), C42-164 (1), L52-115 (C), L136-198 (B), C107-175 (4),

C99-124 (3), L136-198 (A), C114-154 (2)

1 Copie d'ancien 1 poèmes de la dynastie Tang imités par un ignorant (à la mémoire de Bernard Saby)

2 Copie d'ancien 2 en rêvant à l'avenir de la Chine (à la mémoire d'Athanase Kircher)

3 Le Japon côte à côte avec en filigrane des échos des vingt et un classiques en Transit (à la mémoire de Lafcadio Hearn) 4 Copie d'ancien 3 dans la hantise des bouleversements de la Chine (à la mémoire du marquis d'Hervey Saint-Denis) 5 Copie d'ancien 4 en m'efforçant de constituer un talisman qui aide le dragon à se réveiller bienfaisant (à la mémoire de Judith Gautier)

6 Sur la montagne de charbon (à la mémoire de Marco Polo) 717 nuits de Chine avec le souvenir du rossignol qui chantait dans les jardins décrits par le frère Attiret (à la mémoire d'Igor Stravinsky) 8 La Voie des statues, scénario (à la mémoire de Camille Claudel) 
C'est le programme le plus important, ne serait-ce que par le nombre de plages (huit), et la place qu'il occupe. Le seul également à occuper tour à tour chacun des 2 fois 4 canaux. Mais c'est peut-être aussi le programme le plus composite. Il y est question non seulement de la Chine, mais aussi du Japon. Un grand nombre de «sources" sont indiquées dans la table des sources. Enfin, la partie « Copie d'ancien » fait l'objet à elle seule d'une « récapitulation ». Onze poètes chinois de la dynastie des Tang (618-907), période considérée comme l'âge d'or de la poésie chinoise, $y$ sont "imités», dont trois plus particulièrement : Li Bai et son ami Du Fu ainsi que Bai Juyi ${ }^{6}$.

«Le Japon côte à côte »... En 1993, Butor avait publié Tables d'orientation. Au Japon côte à côte. Mais à côté de ce parallélisme des textes (deux sizains par page) qui se calque sur le parallélisme des photographies, un autre parallélisme prend place ici, celui établi par Luis Frois entre Japonais et Européens au XVIe siècle et publié pour la première fois en français l'année même du texte précédent (ces citations entrent elles-mêmes en résonance avec celles du manuscrit d'Alep et du livre de Marco Polo qui courent à la même place, et également en italique, tout le long de «Copie d'ancien »). C'est d'ailleurs dans cette plage qu'un rappel de Transit, où une large place était faite au Japon, s'effectue. Le lecteur est invité à comparer les deux œuvres, dont la disposition des textes dans le volume offre de même un certain parallélisme, mais aussi quelques « contradictions » au sens froisien du terme. Enfin, 1993 est aussi l'année où Butor va pour la première fois en Chine, le Japon constituant de fait une véritable transition vers cette dernière.

6 À titre de comparaison, on pourra mettre en regard les «imitations» butoriennes avec les nombreux poèmes correspondants de ces divers auteurs présents dans Demiéville, 1999. 
Dans le texte "Sur la montagne de charbon", nous tournons avec Segalen (voir Butor, 1999) autour de la Cité interdite à "Pei-king ». En contrepoint, les commentaires du voyageur Butor à Pékin, un Pékin bien différent, bien sûr, de celui de son illustre devancier.

Le texte «17 nuits de Chine» reprend Nuits de Chine (1990). Il se compose également de citations de et autour du frère Attiret, peintre et missionnaire, arrivé en Chine en 1739, de citations de H. C. Andersen et de son «Rossignol » chinois, mais transformées, sinisées pourrait-on dire puisqu'elles imitent les « imitations » précédentes.

«La Voie des statues », scénario, précise le sous-titre. La Voie des statues est en effet un film (1993c) de Jean-Michel Vecchiet avec la collaboration de Michel Butor, tourné à l'occasion de l'exposition Rodin à Pékin. Les deux grands poètes amis, Li Bai et $\mathrm{Du} \mathrm{Fu,} \mathrm{y} \mathrm{continuent} \mathrm{leur} \mathrm{dialogue} \mathrm{au} \mathrm{milieu} \mathrm{de}$ beaucoup d'autres voix qui s'entrecroisent, se prolongent, se superposent, s'entrechoquent.

\section{Pyramide}

\section{L7-51 (C) et C44-94 (2)}

1 Survie Maya (à la mémoire d'Alexandre de Humboldt) 2 Projet Maya (à la mémoire de Guillaume Apollinaire)

Comme le programme Avatar, ce programme ne comprend que deux parties ou «versants »: «Survie Maya » (L7-L51) et « Projet Maya » (C44-C94). 
Ce programme reprend intégralement le texte paru dans Terre maya (1993b) avec des photographies de Marco Dejaegher ${ }^{7}$. Ce texte se présentait dans cet ouvrage sur vingt pages du fait que « chez les Mayas tout allait par vingt, un peu comme chez nos ancêtres les Gaulois » (Gyroscope, "Entrée lettres », p. 25, canal C).

Sa distribution, ici, est reprise sous une forme différente, mais le texte est intégralement conservé. Il contient notamment dix citations du Livre du Conseil, autrement dit le Popol-Vuh, la «Bible» des Mayas, qui ont fait l'objet d'une publication antérieure sous le titre "Selon le livre du conseil ${ }^{8}$, et dix citations d'un autre livre maya, le Livre de Chilam Balam de Chumayel que Benjamin Péret avait traduit et publié en 1955 et auquel s'est intéressé également Le Clézio (2001). Dans le texte ces citations alternent.

Par ailleurs, sous le mot «Aujourd'hui », vingt paragraphes renvoient à certaines photographies du livre. Ce sont donc en principe des paragraphes descriptifs. En réalité, certains paragraphes font allusion à des photographies absentes. L'aspect historique de la civilisation maya est largement développé à travers d'autres paragraphes, de même que l'aspect guide touristique et la part de récit autobiographique.

7 Ce photographe belge est aussi l'auteur d'un autre livre avec Butor: $A u$ Pérou : au pays des Incas... d'aujourd'hui, Lausanne, éditions Livre total, 1990.

8 Texte dédié à Marie Léonor, typographe et calligraphe, fondatrice avec Patrice Pouperon des éditions La Garonne. "Selon le livre du conseil » est également lisible sur le site personnel de l'auteur. 


\section{Minotaure}

L8-24/L24-38 (B); C45-59/C59-77/C79-97 (3) ;

L129-151/L153-171 (D) et C166-200 (1)

1 Picasso-labyrinthe qui comprend :

1 Le bleu des mères (à la mémoire de Claudio Monteverdi)

2 La danse des cornes (à la mémoire du Titien)

2 Picasso-labyrinthe qui comprend :

3 Le cristal des fuites (à la mémoire de Thomas Corneille) 4 Le piège aux femmes (à la mémoire du Tintoret) 5 L'heure de la vérité (à la mémoire de Charles Perrault)

3 Picasso-labyrinthe qui comprend :

6 Le musée des errances (à la mémoire du Corrège) 7 L'ivresse de l'âge (à la mémoire de Hugo von Hofmannsthal)

4 Miroir de Suzanne (à la mémoire de saint Jérôme)

Deux textes occupent ce programme : Picasso-Labyrinthe (PL) et Miroir de Suzanne (MS).

PL est composé de sept sections formant trois ensembles : sections I et II, sections III, IV et V et sections VI et VII. Une des articulations majeures de ce texte est la fin de la cinquième section, qui se termine sur le mot «meurtre » : « À ce mot, le plus fort de tout le texte, noir complet avec une musique violente » (Gyroscope, « Porte chiffres », p. 95, canal 3), précise- 
t-on. Cette section, intitulée « L’heure de la vérité », renvoie à la tauromachie.

Mais il est nécessaire d'explorer plus avant ce premier texte qui se présente, comme nombre d'autres parties de cet ouvrage, comme un conglomérat ou agrégat d'autres textes. Textes publiés antérieurement par l'auteur ou textes venus d'autres horizons.

PL intègre notamment et en tout premier lieu le texte intitulé "Picasso-Labyrinthe, récit de la narratrice», publié en particulier dans le livre de Skimao et Teulon-Nouailles (1988) comme «texte inédit». En réalité, c'est déjà une reprise puisqu'en 1986, était alors paru «Picasso-Labyrinthe, livret pour un film » en version bilingue français-allemand. Ce « récit de la narratrice » apparaît dans ce cinquième Génie du lieu sous la lettre « $\mathrm{N} »$.

Un second texte prend place ici, qui est intitulé «Trois femmes enlacées », texte qui date de 1984 et qui se lit sous la lettre « $\mathrm{C} »(\mathrm{C}$ comme Comparateur).

Un troisième texte se présente ensuite: «La suite dans les images », sous la lettre «E » (E comme Explicateur). Il s'agit d'un texte écrit à l'occasion des 80 ans de Picasso, publié dans Les Lettres françaises en octobre 1961 et repris dans Répertoire III (1968, p. 263-268).

Puis viennent des citations de Picasso, Apollinaire, Balzac et Racine sous la lettre « L » (L comme Lecteur). 
Enfin, le texte «Ballade du sorcier de Mougins », publié dans Liminaires et préliminaires $(1982)^{9}$ et écrit «pour André Villers à la mémoire de Picasso », s'intègre au texte principal à partir de la seconde section.

Le tout couronné par un ensemble de citations de Plutarque/Amyot tirées de l'histoire de Thésée dans Les Vies des hommes illustres.

Soit un ensemble de «textes » qui peuvent se distinguer en trois groupes: les textes de Butor lui-même, les courtes citations d'auteurs français et de larges extraits d'un texte de l'Antiquité grecque.

On dira que tout cela doit former un ensemble bien hétéroclite. Ce à quoi on peut répondre : pas plus que certaines œuvres de Picasso faites avec «de la peinture du papier du charbon de la ficelle et des clous [...] de la tôle de la glaise et de la colle [...] du ciment de la terre de l'osier des feuilles et du plâtre » (Gyroscope, « Entrée lettres », p. 30, canal B).

Quant à l'intégration des textes à l'intérieur de PL, elle présente des caractéristiques différentes selon le texte abordé. Ainsi, le texte de Butor, le plus ancien, «La suite dans les images », n'est pas intégralement repris, mais pour seulement une moitié environ, dont les extraits sont cités dans l'ordre primitif de succession. Il s'agit d'une sorte d'essai sur Picasso qui relaie un peu les analyses de Guillaume Apollinaire dans ses Chroniques d'art, citées par ailleurs dans $\mathrm{PL}^{10}$. On y relève ainsi

\footnotetext{
${ }_{9}$ Cette ballade a été reprise de nombreuses fois : voir Desoubeaux, «Villers, André ».

10 On connaît la célèbre tirade d'Apollinaire (1960, p. 290) sur Picasso dans un article du 14 mars 1913. Citation reprise dans Gyroscope, "Entrée lettres », p. 157, canal D).
} 
que «dans la conscience picturale de Picasso un progrès considérable s'accomplit, [...] il s'agit de l'intégration à l'œuvre d'art de sa propre historicité » (Gyroscope, "Entrée lettres», p. 163, canal D).

Le « Récit de la narratrice », qui mêle étroitement la fable mino-icaro-dédalienne à l'œuvre et à la vie de Picasso, pour sa part, est intégralement repris (avec de légères retouches) comme il le sera de nouveau dans Les Ateliers de Picasso en 2003.

De même, la «Ballade du sorcier de Mougins » est elle aussi intégralement reprise p. L.30, L.32, C.71, C.75, L.131, L.133 et L.147. «L'envoi » étant lu, contrairement au reste du texte, non par «le Diseur», mais par «la Diseuse», le meurtre engendrant en quelque sorte le changement de sexe.

Mais en ce qui concerne les "Trois femmes enlacées", nous assistons à une reprise différente. C'est un texte composé de quinze strophes qui se suivent dans un certain ordre reproduit aussi bien dans la revue Corps écrit, en 1985, que dans Collation, en 1991 ou encore dans l'Anthologie nomade en 2004. Reprise différente tout d'abord parce que le texte n'est pas repris une seule fois comme les textes précédents, mais pas moins de quatre fois dans l'ensemble, non pas de PL, mais du programme tout entier puisqu'en effet, il est en partie repris dans « Miroir de Suzanne».

Il apparaît donc dans son ensemble une première fois dans les sections I, II et III de PL. Il apparaît ensuite deux autres fois dans les sections IV, V et VI et une quatrième fois enfin dans la section VII et MS. Dans sa première version, les strophes ne suivent pas l'ordre du texte isolé de 1 à 15 , mais obéissent au 
schéma suivant : (I) 1, 2, 3, 4 et 5 ; (II) 11, 12, 13, 14 et 15; (III) $6,7,8,9$ et 10 . Ordre au cours duquel on observe une première torsion du texte primitif, torsion accentuée, faut-il le préciser, par le fait que le lecteur doit retourner le volume pour poursuivre sa lecture.

La section IV, quant à elle, reprendra les strophes des première et seconde sections en les enlaçant ou tressant : 1,11 , $2,12,3,13$, 4, etc. La section $\mathrm{V}$, poursuivant cette dynamique, reprend les strophes des seconde (à nouveau) et troisième sections. Et la section VI reprendra à son tour les strophes des première et troisième sections (si bien qu'à l'intérieur de ces trois sections, IV, V et VI, nous avons bien deux fois le reprise du texte dans son intégralité).

Quant à sa quatrième reprise, elle se fait, comme nous l'avons dit, à cheval sur PL et MS. Mais si, dans les quatre premières sections de PL, les strophes sont reprises enlacées plus ou moins mais de façon monolithique, il n'en est pas de même à partir de la cinquième section où, toujours enlacées, elles se disloquent pour s'enlacer davantage les unes les autres. Nous aurons toujours ainsi des strophes de huit vers ou huitains, mais quatre vers seront empruntés à une strophe et quatre vers à une autre, et ce, de façon constante dans cette cinquième section. Dans la sixième, les choses se compliquent encore. Les huitains s'enlacent encore plus puisque nous avons deux vers d'une strophe, puis deux vers d'une autre strophe, puis à nouveau, deux vers de la première et deux vers de la seconde. Ce sont donc deux strophes qui s'enlacent, mais de façon beaucoup plus poussée qu'au début, qui se fondent presque, et ce, de façon constante dans cette sixième section. 
Dans la septième section, enfin, le dispositif n'est plus le même: la partie du texte d'origine appartenant à PL fait toujours apparaître des huitains, mais la partie contenue dans MS se répartit en quatrains. Dans la partie de PL, les huitains sont repris tantôt de façon monolithique (p. L.157, L.159 et L.161), tantôt de façon hétérogène, mélange de deux strophes (p. L.163, L.165 et L.169), tantôt de façon encore plus hétérogène, mélange de trois strophes (p. L.167) et, enfin, de façon entièrement hétérogène, mélange de quatre strophes (p. L.169). Cet ensemble reprend de cette manière l'équivalent de huit strophes (sur quinze) du texte primitif, les sept autres apparaissant sous la forme de quatrains le plus souvent hétérogènes, mélange de deux strophes, dans MS ${ }^{11}$. Ce texte et ce dispositif soulignent par là même la liaison entre les deux parties du programme Minotaure.

Vu sous l'angle du tressage des textes dans leur diversité, de leur disposition sur la page, on peut donc dire que l'adéquation du texte dans sa matérialité-historicité même à son objet - Picasso, dont le caractère hétérogène des œuvres, mais aussi le renouvellement constant de l'inspiration tout au long de sa vie - et aux thèmes que son œuvre et sa vie drainent (tauromachie, femmes, labyrinthe notamment) est particulièrement remarquable et qu'une analyse plus approfondie du texte passe nécessairement par la prise en compte de cette matérialité-historicité propre.

11 Lecteur assidu de la Bible, Butor ne se contente pas d'évoquer un personnage biblique comme Suzanne, il dédie cette quatrième plage du programme à saint Jérôme, traducteur de la Bible en latin. On trouvera également, dans " Miroir de Suzanne », quatorze strophes de la première des Lamentations de Jérémie (La Sainte Bible, p. 1268-1269). Ce sont donc, en l'occurrence, trois niveaux textuels et historiques qui sont pris en considération. 


\section{Vision}

L10-26 (A), C28-42 (2), L116-134 (B), C177-197 (4)

1 La Rose des Voix, premier cercle (à la mémoire de Charles Fourier)

2 La Rose des Voix, deuxième cercle (à la mémoire de Chrétien de Troyes)

3 La Rose des Voix, troisième cercle (à la mémoire de Jean de Meung)

4 La Rose des Voix, quatrième cercle (à la mémoire de Leibniz)

Pour ce programme, je laisse le soin au lecteur de le lire en s'aidant de La Vision de Namur. À l'intention de la rose des voix (1983).

On le voit donc, Gyroscope développe des aspects particuliers liés aussi bien à une ville (Elseneur), un thème scientifique (l'astronomie), un site archéologique (Angkor), un écrivain (Rimbaud), un pays (la Chine), une région touristique (l'Amérique centrale), un peintre (Picasso), une expérience musicale (chœurs de voix), mais qui, tous, prennent appui sur un ou plusieurs lieux qui nous permettent de faire ainsi le tour de la Terre, mais aussi nous amènent à nous plonger et replonger toujours davantage dans la Bibliothèque. Et comme le dit Jean Roudaut (1989, p. 633) à propos du troisième Génie du lieu, Boomerang, «[c]e ne sont plus les aventures d'un narrateur dont le timbre de la voix est effacé, dont les éléments biographiques ne sont plus des événements personnels mais 
des indices généraux, ce ne sont plus les aventures d'un narrateur-voyageur en Amérique ou en Australie qui constituent l'essentiel du récit, mais celles du lecteur qui poursuit le fil du texte de pages roses en pages bleues, de jours en nuits. »

\section{Bibliographie}

ANDERSEN, Hans Christian. 1989, Les Habits neufs de l'Empereur et autres récits, Paris, Flammarion.

ApollinAire, Guillaume. 1960, Chroniques d'art (1902-1918), Paris, Gallimard.

Butor, Michel. 1963, Description de San Marco, Paris, Gallimard; repris dans CEuvres complètes V, Le Génie du lieu 1, Paris, éditions de la différence, 2007.

-. 1968, Répertoire III, Paris, Minuit; repris dans OEuvres complètes, II, Répertoire 1, Paris, éditions de la différence, 2006.

—. 1971, Où, Le Génie du lieu 2, Paris, Gallimard; repris dans OEuvres complètes VI, Le Génie du lieu 2, Paris, éditions de la différence, 2007.

-. 1979, Elseneur, suite dramatique, Yverdon, H. Cornaz, coll. "Volumen » 1. Préface de Roger Francillon. Livret proposé à René Koering à partir d'une idée de Christian Dotremont. Repris dans Avant-goût III, L'appel du large, avant de l'être dans Gyroscope. 
-. 1982, Liminaires et préliminaires, 21 ballades et 7 chansons précédées d'un entretien avec Dominique Bedou, Gourdon, Dominique Bedou éditeur.

- en coll. avec Henri Pousseur. 1983, La Vision de Namur. À l'intention de la rose des voix, Yverdon-les-Bains, Éditions de la Thièle.

—. 1984, Trois femmes enlacées, Genève, Galerie Marie-Louise Jeanneret éditeur; repris dans Corps écrit, $\mathrm{n}^{\circ} 15$, septembre 1985, ainsi que dans Jean-Claude Prêtre, Suzanne, le procès du modèle, Lausanne, La Bibliothèque des Arts, 1990, et dans Collation, Rouen, L'Instant perpétuel éditeur, 1991, sans oublier Anthologie nomade, Paris, Gallimard, 2004.

-. 1986, Picasso-Labyrinthe, livret pour un film = Filmszenario, photographies d'André Villers, texte de Michel Butor, Tübingen, Konkursbuchverlag, C. Gehrke (Tübingen Rive gauche). Édition bilingue, traduction allemande de Helmut Scheffel.

—. 1987, Avant-goût II, Rennes, éditions Ubacs.

-. 1988, Angkor silencieux, photographies de Philippe Gras, textes de Michel Butor et Nouth Narang, Gujan-Mestras, Éditions sous Le Vent.

—. 1989a, Avant-goût III, L'appel du large, Rennes, éditions Ubacs.

—. 1989b, Improvisations sur Rimbaud, Paris, éditions de la Différence.

-. 1989c, Hallucinations simples, voix/textes de Michel Butor et Jean-Marie Le Sidaner, Paris, éditions Artalect. Coffret de deux cassettes audio : I "Hallucinations simples » suivi de «Villas 1900 » et II « Entretiens » avec J.-M. Le Sidaner.

-. 1990, Nuits de Chine : de l'hiver au printemps 1988, photographies de Muriel Olesen, texte de Michel Butor et 
Gérald Minkoff, postface de Charles-Henri Favrod, Genève, édition Miss Understanding for All.

—. 1991, Collation, éclairée par 15 chandeliers dus à l'encre de Michel Sicard, Rouen, L'instant perpétuel éditeur.

—. 1992, Transit, le Génie du lieu, 4, Paris Gallimard; repris dans OEuvres complètes VII, Le Génie du lieu 3, Paris, éditions de la différence, 2008.

-. 1993a, Tables d'orientation. Au Japon côte à côte, photographies de Gérald Minkoff et Muriel Olesen, textes de Michel Butor, Genève, Éditions Olizane.

-. 1993b, Terre maya, photographies de Marco Dejaegher, texte de Michel Butor, Paris, Casterman.

-. 1993c, La Voie des Statues, film de Jean-Michel Vecchiet avec la collaboration de Michel Butor, Ellipse-Elf.

—. 1996, Gyroscope, autrement dit le Génie du lieu, 5 et dernier, Paris, Gallimard; repris dans OEuvres complètes VII, Le Génie du lieu 3 et VIII, Matière de rêves, Paris, éditions de la différence, 2008.

—. 1999, «Au miroir de la Chine », préface à René Leys de Victor Segalen, Paris, éditions Chatelain-Julien.

-. 2003, Les Ateliers de Picasso, Paris, éditions Images modernes [240 pages dont 25 pages de texte de Michel Butor ("L'Alambic des formes»), une biographie, une bibliographie, un index des œuvres, 160 photographies noir et blanc par 18 photographes].

—. 2004, Anthologie nomade, Paris, Gallimard, coll. «Poésie». Préface («L'harmonie des états ») de Frédéric-Yves Jeannet.

—. 2004, L'Horticulteur itinérant, Paris, Léo Scheer, coll. «Melville».

Demiéville. Paul. 1999, Anthologie de la poésie chinoise classique, Paris, Gallimard, coll. « Poésie». 
FONTENELLE, Bernard de. 1724, Entretiens sur la pluralité des mondes, chez Michel Brunet.

FroIS, Luis. 1993, Traité de Luís Fróis, S.J. (1585) : sur les contradictions de mœurs entre Européens et Japonais, traduit $\mathrm{du}$ portugais par Xavier de Castro, Paris, Éditions Chandeigne.

Le ClÉzio, J. M. G. 2001, Les Prophéties du Chilam Balam, Paris, Gallimard, coll. « Le Chemin ».

LuCiEn DE SAmosate. 1973, Histoire véritable, Paris, Gallimard, coll. «Folio ». À noter que les œuvres complètes de cet auteur grec du IIe siècle sont lisibles en français sur la Toile.

PÉRET, Benjamin. 1955, Livre de Chilam Balam de Chumayel, traduit de l'espagnol et présenté par Benjamin Péret, avec 12 illustrations hors-texte et tous les dessins du manuscrit original, Paris, Denoël.

PlutaRque. 1985, Les Vies des hommes illustres, traduction de Jacques Amyot, Paris, Gallimard, coll. « Bibliothèque de la Pléiade ».

RoUDAUT, Jean. 1989, "Récit de voyage », dans Encyclopaedia Universalis, t. 19, p. 630-636. (citation p. 633, 3e col.).

La Sainte Bible. 1955, Paris, Desclée de Brouwer.

SKIMAO et Bernard Teulon-Nouailles. 1988, Qui êtes-vous Michel Butor?, Paris, La Manufacture.

VERnE, Jules. 1966, Autour de la Lune, Paris, Librairie Hachette. 


\title{
Résumé
}

Toute lecture de Gyroscope, livre-labyrinthe s'il en est, nécessite, pour en faire une analyse plus poussée, un premier déchiffrement des divers éléments que sont les montagestextes qui le composent. C'est à cette tâche que cet article s'emploie. À la lueur de ce premier travail, il apparaît que de très nombreuses citations, autocitations, réécritures, répétitions, reprises, "imitations", variations, se combinant avec la physique particulière de l'ouvrage, sont partout à l'œuvre. En même temps qu'à un voyage tout autour de la Terre, c'est à un voyage à travers la Bibliothèque que le lecteur est convié.

\begin{abstract}
Any reading of Gyroscope, a maze-book, is incomplete without a first overview of the different literary building-blocks on which this work is fashioned. Any researcher should probably know this overview before carrying out a sound analysis. The following article is designed to describe this process. This first assignment shows that the work itself is constructed around numerous quotations, auto-quotations, re-writings, reworkings, "imitations" and variations. The reader soon realizes that this work is both a voyage around the Earth and a trip through a library.
\end{abstract}

